\title{
A Note on the Effect of the Free Surface on the Elastic Deformation due to a Long Dip-Slip Fault
}

\author{
Sarva Jit Singh* and Mukesh Punia \\ Department of Mathematics, Maharshi Dayanand University, \\ Rohtak-124001, India
}

\begin{abstract}
To calculate the elastic deformation due to a thrust fault in the lithosphereasthenosphere composite, Nur and Mavko (1974) used the exact solution of Mura (1968) for an edge dislocation parallel to the boundary of two elastic half-spaces in welded contact. They approximated the effect of the free surface by considering Mura's solution for two equal and opposite edge dislocations with the free surface midway between them. By considering a simpler problem of a long dip-slip fault in a uniform half-space, we show that the approximate method used by Nur and Mavko does not yield satisfactory results. For small dip angles and beyond a certain epicentral distance, while the exact solution predicts subsidence, the approximate solution predicts uplift. Moreover, the approximate solution yields non-zero values for the surface tractions at the free surface.
\end{abstract}

\section{Introduction}

Two-dimensional dip-slip dislocation models have been used extensively to model the crustal deformation associated with thrust faulting at subduction zones. In the analytical approach, one must solve the problem of a long dip-slip fault in a layer (representing the lithosphere) lying over a half-space (representing the asthenosphere). However, the analytical expressions for the displacements and stresses for such a model are complex and are not amenable to straightforward numerical computation. To circumvent this difficulty, Nur and Mavko (1974) began with the exact solution for an edge dislocation parallel to the boundary of two welded elastic half-spaces given by Mura (1968). The effect of the free surface was taken into account by them approximately by using Mura's solution for two equal and opposite edge dislocations with the free surface midway between the two dislocations. There seems to be no justification for the approximate method used by Nur and Mavko (1974). To examine this, we consider a simpler problem; i.e., the problem of a long dip-slip fault in a uniform half-space. We show that the approximate method of Nur and Mavko (1974), as applied to this simpler model, does not give satisfactory results. For small dip angles (including a dip of $30^{\circ}$ considered by Nur and Mavko) and beyond a certain epicentral distance, while the exact solution predicts subsidence, the approximate method predicts uplift.

Received August 23, 1993; Accepted March 8, 1994

* To whom correspondence should be addressed. 
Moreover, the approximate method yields non-zero values for the surface tractions $p_{23}$ and $p_{33}$ at the free surface $\left(x_{3}=0\right)$. These tractions vanish at the free surface in the exact solution due to the boundary conditions.

\section{Theory}

Rani and Singh (1992) derived closed-form expressions for the displacements and stresses at an arbitrary point of a homogeneous, isotropic, perfectly elastic half-space caused by a long dip-slip fault parallel to the surface. Let the Cartesian coordinates be denoted by $\left(x_{1}, x_{2}, x_{3}\right)$ with the $x_{3}$-axis vertically downwards and the half-space occupying the region $x_{3}>0$. Then the expressions for the surface displacements and stresses for reverse slip (thrust fault) of amount $b$ along the down-dip segment from the surface to a distance $L$ are

$$
\begin{gathered}
U_{2}=\frac{1}{\pi}\left[\cos \delta\left\{\tan ^{-1}\left(\frac{Y-\cos \delta}{\sin \delta}\right) \mp \frac{\pi}{2}\right\}+\frac{\sin \delta}{A^{2}}(1-Y \cos \delta)\right], \\
U_{3}=\frac{1}{\pi}\left[\sin \delta\left\{\tan ^{-1}\left(\frac{Y-\cos \delta}{\sin \delta}\right) \mp \frac{\pi}{2}\right\}+\frac{Y \sin ^{2} \delta}{A^{2}}\right], \\
P_{22}=\frac{16 \sin \delta}{3 \pi A^{2}}\left(\cos \delta-\frac{Y \sin ^{2} \delta}{A^{2}}\right), \\
P_{23}=P_{33}=0,
\end{gathered}
$$

where $\delta$ denotes the dip angle, $\mu$ the rigidity of the medium and

$$
\begin{gathered}
U_{i}=u_{i} / b, \quad P_{i j}=(L / \mu b) p_{i j}, \\
Y=x_{2} / L, \quad A^{2}=(Y-\cos \delta)^{2}+\sin ^{2} \delta .
\end{gathered}
$$

In the expressions for the displacements, the upper sign (-) is for $Y>0$ and the lower sign $(+)$ is for $Y<0$. All the results are given for a Poissonian material.

Let the expressions for the surface displacements and stresses obtained on using the approximate method of Nur and Mavko (1974) be denoted by $u_{2}^{*}, u_{3}^{*}, p_{22}^{*}, p_{23}^{*}$, and $p_{33}^{*}$. These approximate expressions are obtained on superimposing the solution for the fault in an unbounded medium and the solution for an image fault (with equal and opposite slip) with the free surface midway between the two faults. From Rani and Singh (1992), we obtain

$$
\begin{gathered}
U_{2}^{*}=\frac{1}{\pi}\left[\cos \delta\left\{\tan ^{-1}\left(\frac{Y-\cos \delta}{\sin \delta}\right) \mp \frac{\pi}{2}\right\}-\frac{1}{3 A^{2}} \sin 2 \delta(Y-\cos \delta)\right], \\
U_{3}^{*}=\frac{1}{\pi}\left[\sin \delta\left\{\tan ^{-1}\left(\frac{Y-\cos \delta}{\sin \delta}\right) \mp \frac{\pi}{2}\right\}+\frac{2}{3 A^{2}} \sin ^{2} \delta(Y-\cos \delta)\right], \\
P_{22}^{*}=\frac{2 \sin 2 \delta}{3 \pi A^{2}}\left(3-\frac{2 \sin ^{2} \delta}{A^{2}}\right),
\end{gathered}
$$




$$
\begin{gathered}
P_{23}^{*}=\frac{4 \sin ^{2} \delta}{3 \pi A^{2}}\left[1-\frac{2}{A^{2}}(Y-\cos \delta)^{2}\right], \\
P_{33}^{*}=\frac{2 \sin 2 \delta}{3 \pi A^{2}}\left(-1+\frac{2 \sin ^{2} \delta}{A^{2}}\right) .
\end{gathered}
$$

\section{Numerical Results and Conclusions}

Figure 1 shows the variation of the displacements with the distance from the fault for $\delta=15^{\circ}$. We notice that $U_{2}^{*}$ is approximately equal to $U_{2}$. However, the behaviour of $U_{3}^{*}$ is significantly different from that of $U_{3}$ on the hanging wall side. For $0<x_{2}<0.79 L$, both $U_{3}^{*}$ and $U_{3}$ are of the same sign, but there is a considerable difference in their magnitudes. For $x_{2}>0.79 L, U_{3}^{*}$ and $U_{3}$ are of different signs. Figure 2 is for $\delta=30^{\circ}$. In this case, $U_{2}^{*}$ and $U_{2}$ differ significantly near the fault. $U_{3}$ vanishes for $x_{2}=0.82 L$. The variation of the displacements for $\delta=60^{\circ}$ is shown in Fig. 3. In this case $U_{3}$ vanishes for $x_{2}=1.35 \mathrm{~L}$. For a vertical fault, $U_{2}^{*}$ vanishes identically, but $U_{2}$ is non-zero. $U_{3}^{*}$ and $U_{3}$ show a similar behaviour (Fig. 4). The variation of the stresses with the distance from the fault is shown in Figs. 5-8. We note that though $P_{23}$ and $P_{33}$ vanish identically on account of the boundary conditions at the free surface, $P_{23}^{*}$ and $P_{33}^{*}$ are non-zero for all dip-angles $\left(P_{33}^{*}=0\right.$ for $\left.\delta=90^{\circ}\right)$. Even $P_{22}^{*}$ and $P_{22} \operatorname{differ}$

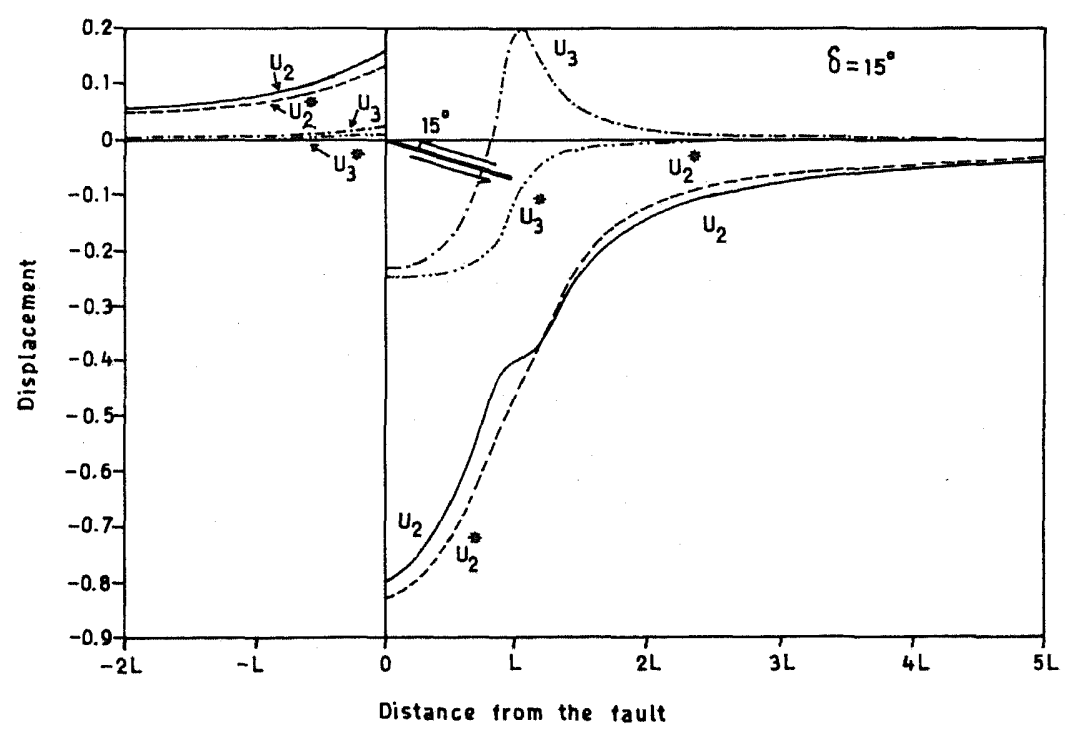

Fig. 1. Variation of the horizontal $\left(U_{2}\right)$ and vertical $\left(U_{3}\right.$, down) surface displacements with the distance from the fault for $\delta=15^{\circ}$. A thrust fault having a slip of amount $b$ along the down-dip segment from the surface to a distance $L$ is situated in a uniform half-space. The displacements are measured in units of the slip $b$ on the fault. $U_{2}^{*}, U_{3}^{*}$ are the displacements for the approximate solution. 


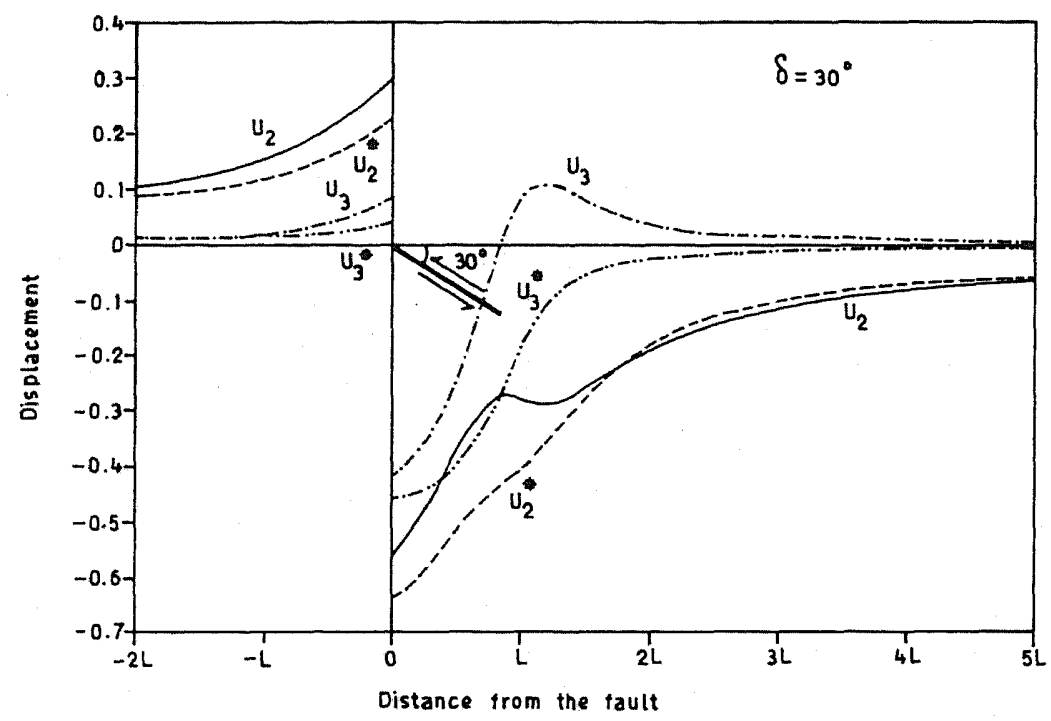

Fig. 2. Same as in Fig. 1 with $\delta=30^{\circ}$.

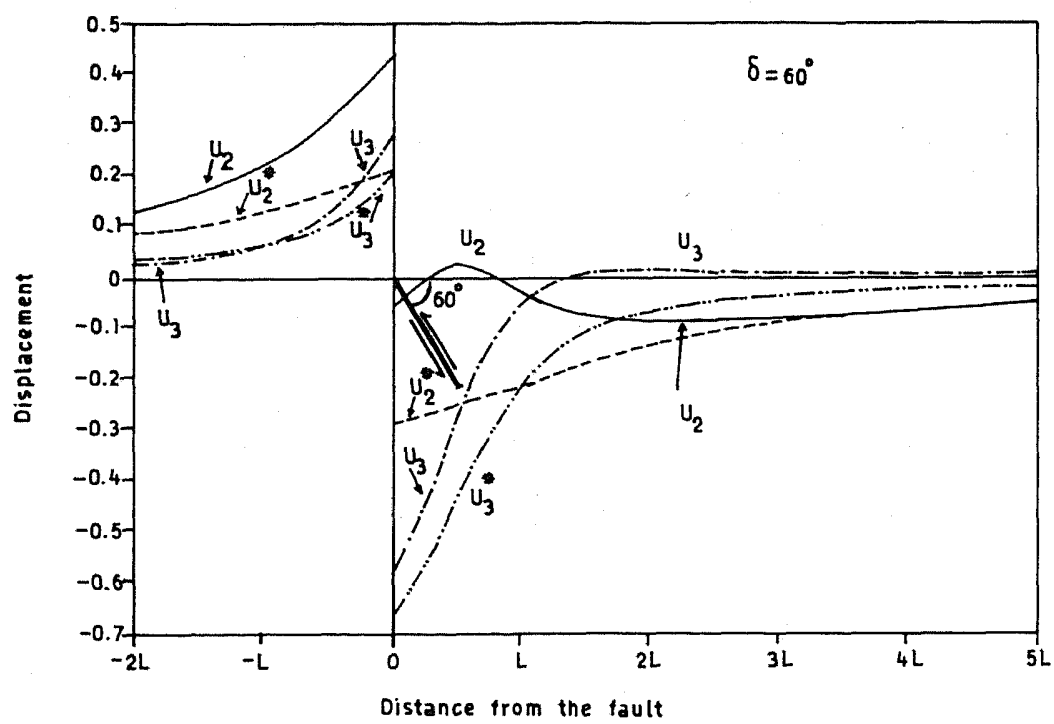

Fig. 3. Same as in Fig. 1 with $\delta=60^{\circ}$. 


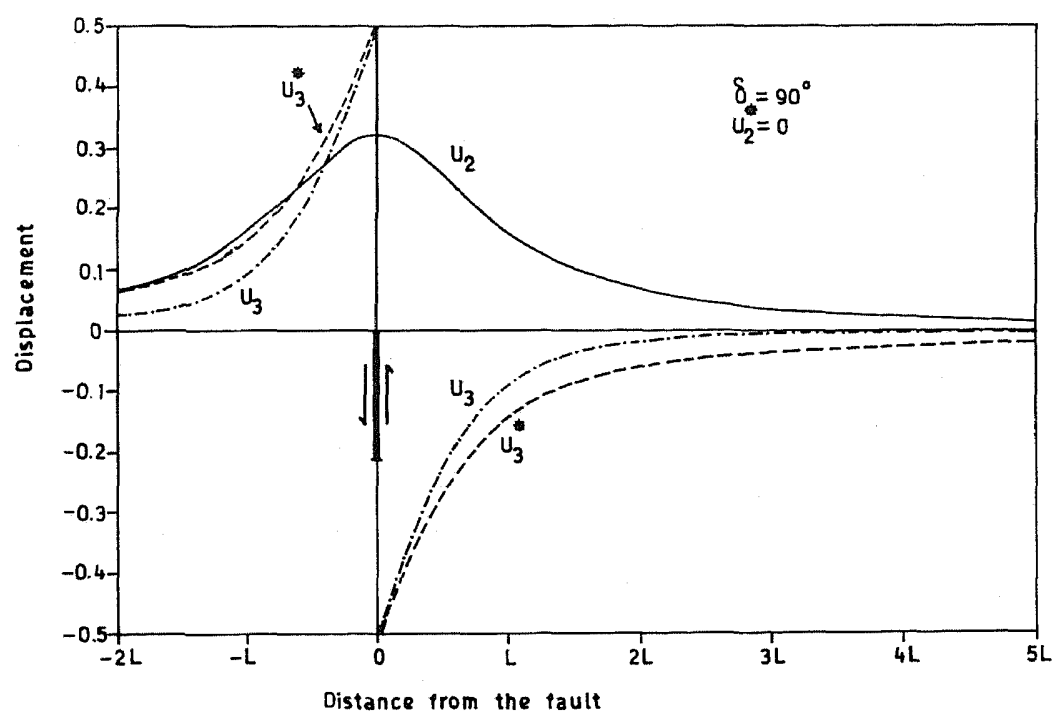

Fig. 4. Same as in Fig. 1 with $\delta=90^{\circ}$.

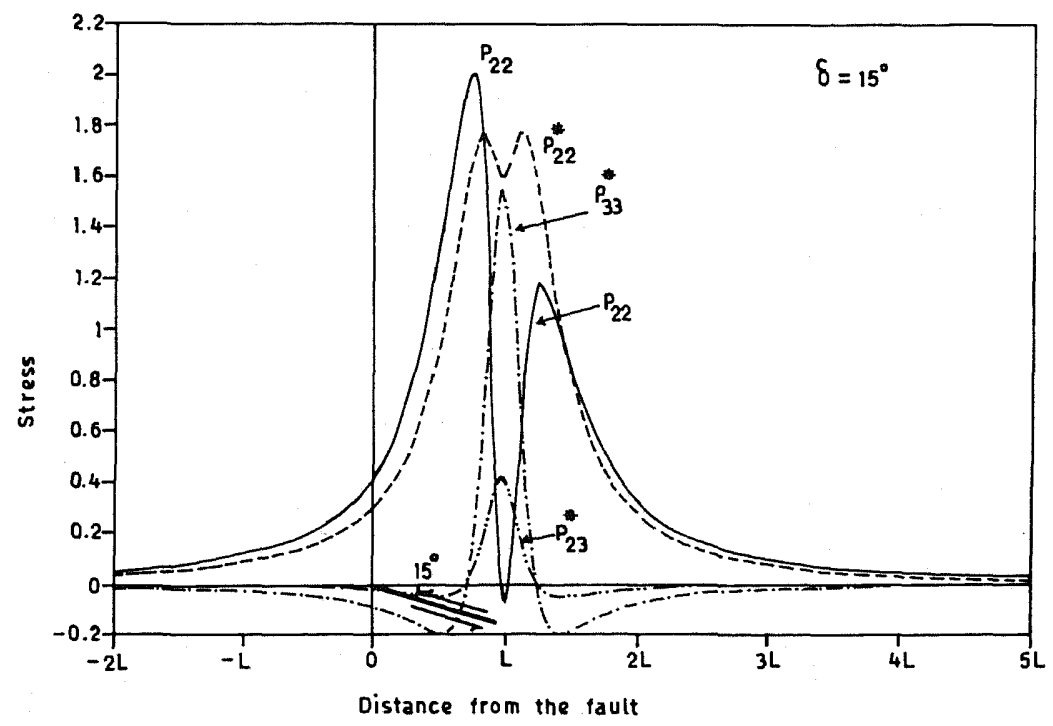

Fig. 5. Variation of the stresses with the distance from the fault for $\delta=15^{\circ}$. The stresses are measured in units of $(\mu b / L) . P_{22}^{*}, P_{23}^{*}, P_{33}^{*}$ are the stresses corresponding to the approximate solution. 


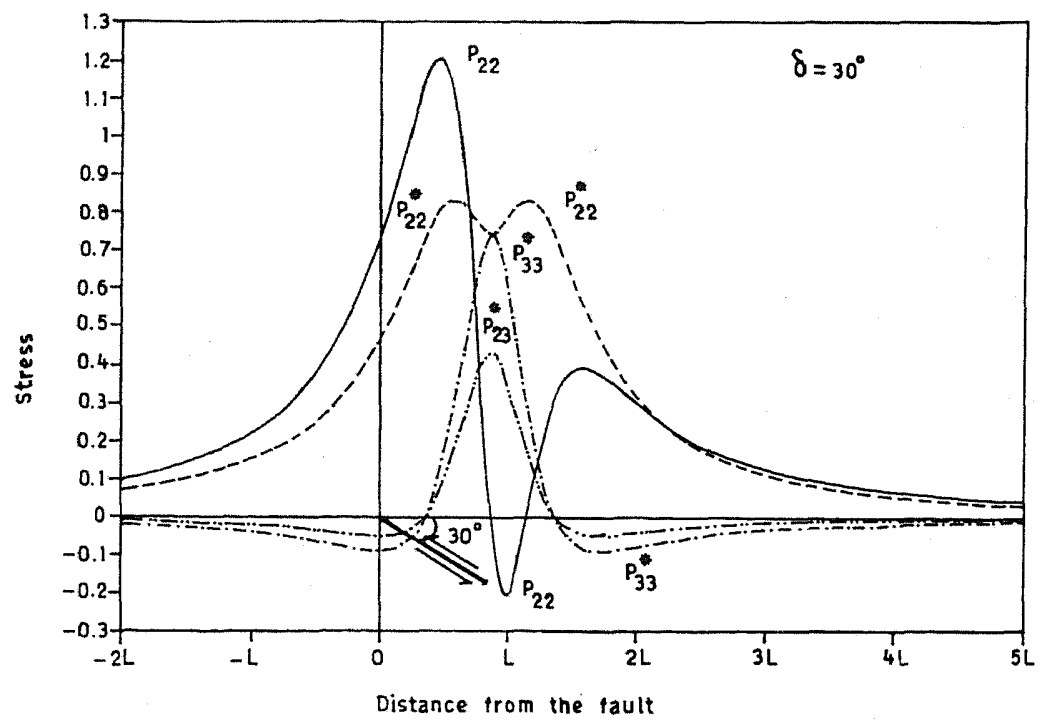

Fig. 6. Same as in Fig. 5 with $\delta=30^{\circ}$.

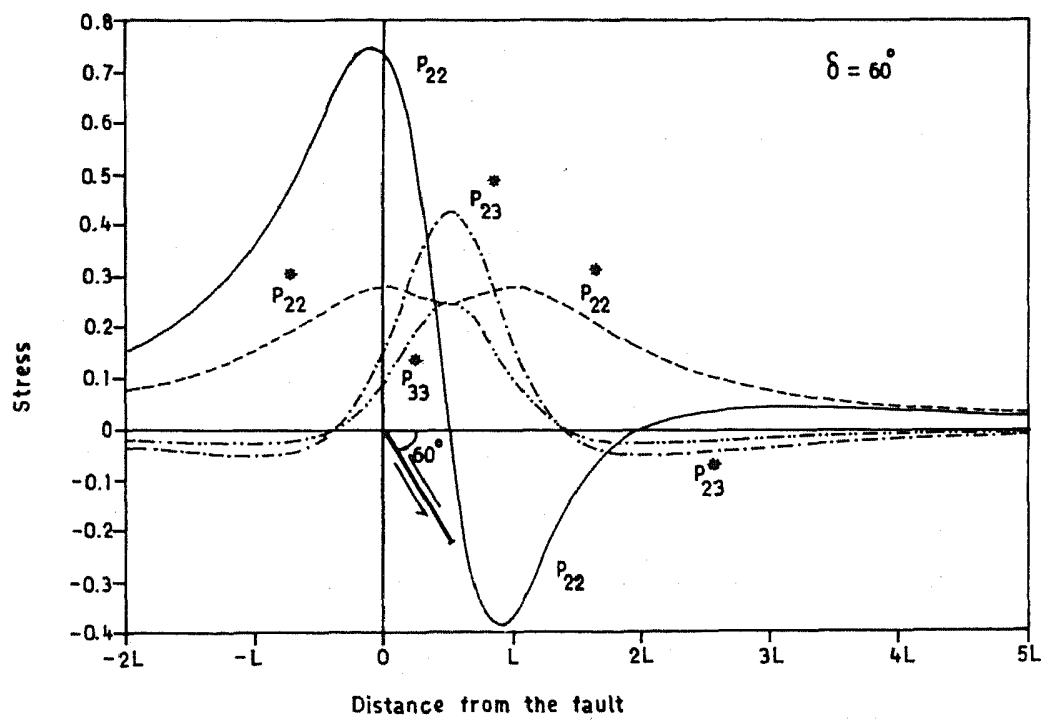

Fig. 7. Same as in Fig. 5 with $\delta=60^{\circ}$. 


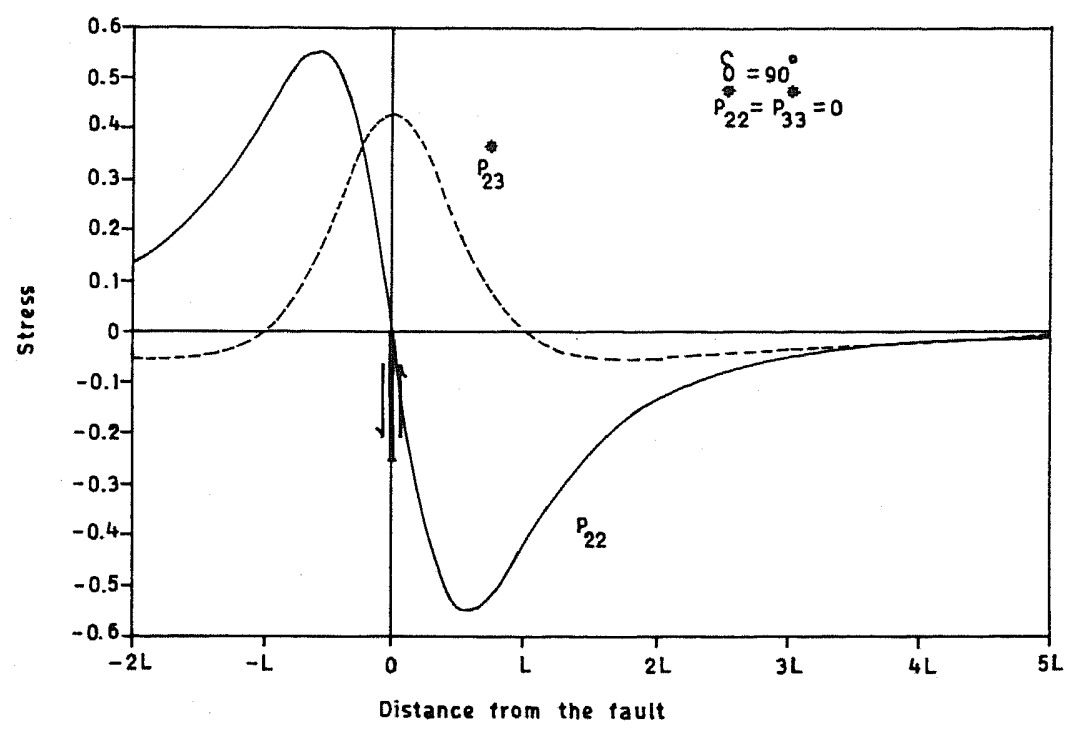

Fig. 8. Same as in Fig. 5 with $\delta=90^{\circ}$.

significantly. In a certain range of values of the distance from the fault, $P_{22}^{*}$ and $P_{22}$ differ in sign as well. It thus appears that the approximate method used by Nur and Mavko (1974) for obtaining the elastic deformation due to a thrust fault in the lithosphere-asthenosphere composite may not be valid.

We are grateful to the Council of Scientific and Industrial Research, New Delhi, for financial support.

\section{REFERENCES}

Mura, T., The continuum theory of dislocations, in Advances in Materials Research, Vol. 3, ed.

H. Herman, pp. 1-108, Wiley, New York, 1968.

Nur, A. and G. Mavko, Postseismic viscoelastic rebound, Science, 183, 204-206, 1974.

Rani, S. and S. J. Singh, Static deformation of a uniform half-space due to a long dip-slip fault, Geophys. J. Int., 109, 469-476, 1992.

Vol. 42, No. 1, 1994 